Pedagogía y Saberes n. ${ }^{\circ} 53$ Universidad Pedagógica Nacional Facultad de Educación. 2020. pp. 133-148

\title{
Un sentido formativo implícito en la Educación Física: el rendimiento*
}

\section{Artículo de investigación}

\section{A Implicit Formative Sense in the Physical Education: The Perfomance}

Um sentido formativo implícito na Educação Física: O rendimento

Daniel Hincapié Bedoya**

Para citar este artículo:

Hincapié, D. (2020). Un sentido formativo implícito en la Educación Física: el rendimiento. Pedagogía y Saberes, 53, 133-148. https://doi.org/10.17227/pys.num53-10574

* Artículo resultado de investigación finalizada de la tesis de maestría "Sentidos formativos implícitos en el saber pedagógico del profesorado en formación de Educación Física”, del programa Motricidad y Desarrollo Humano de la Universidad de Antioquia, asociada al macroproyecto de investigación: “Capacidades docentes y formación de profesionales del campo de la Educación Física, el deporte, la recreación y la actividad física en Medellín”, proyecto aprobado en la convocatoria interna CODI número 2016-13247 de la Universidad de Antioquia. La investigación fue realizada por el grupo Prácticas Corporales, Sociedad, Educación - Currículo (PES).

** Licenciado en Educación Física y maestrando en Motricidad y Desarrollo Humano (tesis en evaluación) de la Universidad de Antioquia (Medellín, Colombia). Docente de cátedra de la Universidad de Antioquia (Medellín, Colombia). Integrante del grupo de investigación Prácticas Corporales, Sociedad, Educación - Currículo (PES). Docente del Colegio Colombo Francés. La Estrella, Antioquia (Colombia).

Correo electrónico: daniel.hincapieb@udea.edu.co

Código Orcid: https://orcid.org/0000-0001-8044-4311 


\title{
Resumen
}

Este artículo es un producto asociado a la investigación "Sentidos formativos implícitos en el saber pedagógico del profesorado en formación de Educación Física" que forma parte de la Maestría en Motricidad y Desarrollo Humano de la Universidad de Antioquia. En ella se presenta el caso del rendimiento, como uno de sus resultados, entendido como lógica educativa fundamentada en la eficiencia y la productividad. Se utilizaron entrevistas semi estructuradas, audiencias con los profesores en formación, observaciones de clase y grabación de videos de clase. Se parte de una contextualización problemática en la que la pedagogía, el maestro y la enseñanza son sistemáticamente desplazados por una discursividad educativa justificada por las presiones del aprendizaje. A partir de un enfoque cualitativo, se practica una metodología soportada por la analítica interpretativa en la que se combinan diferentes modelos de análisis como el posestructuralismo educativo y el análisis de discurso sustentados sobre la base de la teoría fundamentada. Como conclusiones, se advierte el rendimiento como un sentido formativo reconocido en las intencionalidades de los profesores observados y como una orientación captada, implícitamente, en el juego de significados que circulan alrededor de intereses técnicos, de una alta valoración por el saber disciplinar y del predominio de metodologías tradicionales en cuanto elementos constitutivos y configuradores del saber pedagógico de los profesores en formación.

\section{Palabras clave}

formación; educación física; pedagogía; rendimiento

\begin{abstract}
This article is a product associated with the research "Implicit formative senses in the pedagogical knowledge of the teachers of Physical Education in formation" which is part of the Master's Degree in Motricity and Human Development from the University of Antioquia. It presents the case of perfomance as one of its research results, understood as educational logic based on efficiency and productivity. Semi-structured interviews, audiences with the teachers in training, class observations and recordings of class videos were used. It is based on a problematic contextualization where the pedagogy, the teacher and teaching are systematically displaced by an educational discursivity justified by the pressures of learning. Based on a qualitative approach, is practiced a methodology supported by an analytical interpretation, which combining different research methods such as educational poststructuralism and discourse analysis supported on the basis of grounded theory. As conclusions, the performance is recognized as a formative sense in the intentions of the teachers observed and as a orientation captured, implicitly, in the play of meanings circulating around technical interests, a high value for disciplinary knowledge and the predominance of traditional methodologies as constitutive and configurator elements of the pedagogical knowledge of teachers in formation.
\end{abstract}

\section{Keywords}

formation; physical education; pedagogy; performance

\begin{abstract}
Resumo
Este artigo é um produto associado à pesquisa "Sentidos formativos implícitos no saber pedagógico do corpo docente em formação de Educação Física" que faz parte do Mestrado em Motricidade e Desenvolvimento Humano da Universidade de Antioquia. Nela se apresenta o caso do rendimento como um dos seus resultados de pesquisa, entendido como lógica educacional baseada em eficiência e produtividade. Foram utilizadas entrevistas semiestruturadas, conversas com os professores em treinamento, observações em sala de aula e gravação de vídeos. Parte-se de uma contextualização problemática em que a pedagogia, o professor e o ensino são sistematicamente deslocados por uma discursividade educativa justificada pelas pressões da aprendizagem. A partir de uma abordagem qualitativa, pratica-se uma metodologia suportada pela analítica interpretativa na qual se combinam diferentes métodos de pesquisa como pós-estruturalismo educacional e análise de discurso sustentados sobre a teoria fundamentada. Como conclusões, o rendimento é notado como um sentido formativo reconhecido nas intencionalidades dos professores observados e como uma orientação captada, implicitamente, no jogo de significados que circulam em torno de interesses técnicos, de uma alta valorização pelo saber disciplinar e pelo predomínio de metodologias tradicionais enquanto elementos constitutivos e configuradores do saber pedagógico dos professores em formação.
\end{abstract}

\section{Palavras chave}




\section{Introducción}

Este artículo focaliza la reflexión entorno a uno de los hallazgos de la tesis de maestría que tiene entre sus objetivos reconocer los sentidos formativos implícitos en las practicas pedagógicas del profesorado en formación de Educación Física (EF). Se tomó como sujeto de investigación al profesorado en formación de la disciplina de tres universidades analizado en los procesos de intervención de sus prácticas pedagógicas profesionales en el contexto escolar. A su vez, la investigación se encuentra articulada al macroproyecto de investigación "Capacidades docentes y formación de profesionales del campo de la Educación Física, el deporte, la recreación y la actividad física en Medellín", investigación aprobada en la convocatoria interna (CODI número 2016-13247) de la Universidad de Antioquia, realizada por el grupo de investigación PES (Prácticas Corporales, Sociedad, EducaciónCurrículo) de la misma institución, y desarrollado en colaboración con la Universidad San Buenaventura, la Universidad Católica Luis Amigó y el Politécnico Colombiano Jaime Isaza Cadavid. En cuanto a las universidades encargadas de la formación de los profesionales del campo de la EF en el área metropolitana del Valle de Aburrá (Antioquia), investigación desarrollada con la colaboración y la participación de la Universidad de Valladolid (España), a partir de la mediación de un convenio institucional para la participación y colaboración investigativa.

La pesquisa analiza el contexto de la formación profesional del profesorado en relación con los consecuentes procesos pedagógicos en la educación escolar, a partir de la apropiación del saber pedagógico por parte del profesorado en formación de EF. En el análisis se problematizan asuntos como el contexto de una progresiva despedagogización educativa, el desprecio o subvaloración de la enseñanza, la invisibilidad del maestro y la despolitización de la educación; a partir de marcos normativos y epistemológicos. Cuestiones a través de las cuales se advierte una discursividad de la que no parece existir la reflexión necesaria, para el establecimiento de un diálogo crítico en los procesos de formación del profesorado y en los consecuentes espacios y contextos de intervención pedagógica.

En ese sentido, se reconoce una agenda o política educativa de alcances globales desplegada por organismos internacionales, especialmente del ámbito económico, como Unesco, Organización de Estados Americanos (oEA), Programa de Promoción de la Reforma Educativa en América Latina y el Caribe (Preal), la Organización de Estados Iberoamericanos para la Educación, la Ciencia y la Cultura (OEI), el Banco Mundial (BM) y Comisión Económica para
América Latina y el Caribe (Cepal), entre otros; en la que se proyecta la definición de unos marcos comunes para la formación del profesorado. Estas regularidades y regulaciones presentan unas formaciones discursivas generadas, tanto en el campo mundial de la formación de maestros como en la educación, con incidencia en las políticas públicas de los países latinoamericanos. Discurso que ingresa a través de los procesos de acreditación de los programas de formación del profesorado y en la que se reconoce un cambio enunciativo afirmado en conceptos como "[...] desarrollo económico, calidad, alta calidad, descentralización, evaluación, innovación, educación para el trabajo, pruebas internacionales, educación para todos, aprender a lo largo de la vida, emprendimiento, gestión, competencias" (Herrera, 2017).

A lo anterior se suma el relativismo y la ambigüedad presente en los términos legales de la formación del profesorado en Colombia en cuanto a política educativa, en la cual se reconoce una tensión y una contradicción entre las definiciones dadas por la Ley General de Educación Colombiana (Ley 115 de 1994) y el Decreto 1278 de profesionalización docente expedido en 2002. Por un lado, se observan unas definiciones dadas en la Ley 115/94 en las que se establecen las condiciones de una formación pedagógica de base como requisito para la vinculación y el ejercicio profesional docente. Mientras que, por otro lado, el estatuto 1278 de 2002 define unas nuevas condiciones a partir de las cuales la formación pedagógica se ve desplazada o remplazada por la formación disciplinar o la profesionalización dentro de un área o disciplina del conocimiento, lo que permite la incorporación a la carrera docente de profesionales alejados de una formación pedagógica inicial.

En el terreno de lo epistemológico se observa, de igual modo, una creciente despedagogización de la educación. Específicamente, se reconoce una referencialidad centrada en explicaciones científicas y progresistas, en donde el aprendizaje es tomado como factor de desarrollo sustentado por un auge de críticas a partir de las cuales se cuestiona la centralidad del maestro y se define una desvalorización de la enseñanza, cuestión que, según Loureiro y Lopes (2019), modifica la noción de un aprendizaje en términos de formación, por una idea de adquisición de habilidades y competencias para la autogestión, condiciones demandadas por la racionalidad neoliberal, la dinámica del consumo y la inestabilidad laboral. Se advierte una referencialidad en la que se reconoce una forma de desaparición del maestro y de la enseñanza, a partir de la denominada aprendificación, en cuanto nuevo lenguaje asociado a la educación, que parte de la claridad de que el lenguaje del aprendizaje 
es totalmente opuesto al de la educación, en la medida en que el eje del lenguaje educativo no solo obedece a que los estudiantes aprendan, sino a que "aprendan algo", "para un propósito" y "que lo aprendan de alguien", distinto al eje del aprendizaje, que hace más difícil o imposible las preguntas decisivas acerca del "contenido", "el propósito" y "las relaciones" (Biesta, 2016. p. 4).

De esta manera, se reconoce un movimiento discursivo en el terreno educativo cercano a una orientación o enfoque técnico, cuestión que Carr y Kemis (1998) proponen como una educación neutra u objetiva y despojada de su carácter político, ideológico y filosófico (como se citó en Martos-García, Lorente-Catalán y Marínez, 2018). Este enfoque, dada la tradicional constitución técnica y la cercanía de la EF con matrices biomédicas, definen una tensión en el interior de la disciplina a partir de la presencia de discursos que se mueven, entre la determinación de una orientación científica y la constitución de una disposición pedagógica de la EF con efecto en los procesos de formación del profesorado (Tinning, 1996). Lo anterior sugiere la necesidad de un detenimiento reflexivo sobre la apropiación del saber pedagógico por parte del profesorado y de sus intencionalidades formativas, a partir de los ejercicios de saber desplegados en los procesos de enseñanza de la EF en la escuela. En atención a los planteamientos de Carr (1990) y Stenhouse (1987), el desarrollo curricular y su consecuente apropiación pedagógica dependen del desarrollo profesional docente, cuestión que advierte acerca de la necesaria investigación sobre el profesorado.

De igual modo, se observa la emergencia de una discursividad circulando en el ámbito sociocultural y asociada con lo que se ha señalado como una referencialidad posmoderna, en la que se reconoce una narrativa emparentada con códigos propios del campo de la EF, entre los que se cuentan: el protagonismo del cuerpo, el hedonismo, la salud, el cuidado, la ejercitación, la imagen, la nutrición, entre otras preocupaciones que, de acuerdo con Rojas (2000) obedecen a una cultura light proveniente de la práctica del ejercicio físico. Poblete y Gamboa (2013) señalan, en esta misma dirección, que el mundo posmoderno, en el que circula la educación en general y la EF en particular, está impregnado por sociedades y culturas que vienen siendo eje de las concepciones y funciones de la actividad física en general. Este contexto pone en cuestión muchas de las habilidades de los docentes, sobre las cuales no se conocen consecuencias para la EF (Fernández-Balboa, 2003). Guervilla (1993, citado por Gracia, 2008) advierte que esta realidad (posmoderna) no puede descartarse del currículo oficial de la EF, situación que, según Gracia (2008), exige al mismo tiempo la labor del profesorado, como agentes, no solo mediadores sino transformadores de la cultura.
Lo que se descubre es la presencia de una formación discursiva tanto a nivel sociocultural como normativo y epistemológico, en la que la pedagogía, entendida como disciplina a partir de la cual se conceptualiza y se reflexiona sobre la educación y la enseñanza, puede ser desplazada por disposiciones en las que se pasa de la intersubjetividad de los procesos formativos escolares, a los afanes objetivos dados desde la justificación de las competencias y el aprendizaje. Esta cuestión exige reconocer la trama de significados, en términos de sentidos formativos, desplegados a partir del saber pedagógico del profesorado de EF en formación en las intervenciones pedagógicas. De acuerdo con Aguayo (2010), las prácticas escolares desplegadas por el profesorado de EF representan una oportunidad para reconocer el devenir histórico de la educación corporal, pues estas se hayan permeadas por diferentes formas del pensamiento (creencias, valores, prejuicios, ideales, concepciones, imaginarios...) que, en síntesis, retratan las tradiciones que el profesorado incorpora, reproduce y socializa en los patios de las escuelas.

El artículo presenta, en términos de hallazgo investigativo, el sentido formativo del rendimiento, entendido como disposición o intencionalidad formativa implícita en las prácticas de saber pedagógico de los profesores en formación de EF. Gracias a los análisis se reconoce la conexión de dicho sentido, como una orientación que se ve condicionada por las formaciones discursivas presentes en las demandas sociales, el campo epistemológico y los marcos normativos de la formación del profesorado.

\section{Marco teórico conceptual}

En este estudio, la pedagogía aparece posicionada como un saber en la medida en que corresponde a una disciplina encargada tanto de la reflexión teórica de y sobre la práctica de enseñanza, así como de la aplicabilidad práctica en los procesos educativos del ser humano; cuestión que permite reconocer su carácter como disciplina a partir de la cual se conceptualiza, aplica y experimenta lo concerniente a la enseñanza de la cultura y en las culturas (Zuluaga et al., 1988). A su vez, Zuluaga (1999, citado por Zapata, 2003) plantea, a partir de la teorización suministrada por la ruta arqueológica y genealógica, que el saber pedagógico obedece a un campo de conocimiento centrado en la enseñanza y que el sujeto portador de este saber es el maestro, por cuanto corresponde a un dominio propio, producto de la experiencia posibilitada por la práctica pedagógica.

La investigación se fundamenta teórica y epistemológicamente en los campos de estudio educativo y curricular, a partir de una perspectiva filosófica que trata el proceso educativo como una práctica vincu- 
lada a la experiencia y posibilitada por la perspectiva de los actores, en este caso de los profesores en formación. De acuerdo con Díaz (2003), esta elección representa una diferenciación entre dos vertientes o fronteras en las investigaciones curriculares; por un lado, aquellas dimensionadas en el plano de los programas educativos y, por otro lado, una creciente investigación educativa concentrada en la experiencia dentro de los procesos educativos. Desde este marco, se analizan los dominios del maestro, en cuanto sujeto de saber pedagógico, bajo una mirada comprensiva sobre sus intenciones formativas con las prácticas de saber pedgógico; a través del análisis acerca de los tratamientos didácticos, las selecciones curriculares, disciplinares y pedagógicas, y desde los significados y sentidos proyectados desde su práctica de saber, en cuanto maestro y actor del proceso educativo.

El saber pedagógico permite unificar con sentido la diversidad de conocimientos, prácticas y preceptos formativos que, desde los discursos y las instituciones sociales, así como desde las producciones conceptuales de las áreas del conocimiento, se instalan en los procesos de enseñanza a través de la práctica pedagógica (Zapata, 2003) y, a partir de la mediación/intervención del maestro en sus procesos de enseñanza. Es el maestro quien se encarga de poner en contexto formativo la intersubjetividad de los diferentes saberes, a partir de la relación escuela/ sociedad/Estado/cultura (Zuluaga et al., 1988), y es el saber pedagógico, dadas sus posibilidades experienciales y de reflexión sobre la práctica de la enseñanza, el que permite pensar el fenómeno educativo desde adentro, diferente al acercamiento o mirada extraña y exterior dirigida desde las ciencias de la educación sobre la práctica pedagógica (Zapata, 2003).

A su vez, la investigación discute la emergencia del paradigma del aprendizaje en virtud de la posible despedagogización de la educación, trama que arrastra consigo consecuencias en cuanto a la función social de la educación y del maestro, y desde la cual se evidencia una propensión por su despolitización (Biesta, 2016). La discursividad presente, en términos de habilidades y conocimientos relacionados con las presiones de los poderes económicos del actual sistema capitalista, advierte sobre la definición de un aprendizaje relacionado con categorías como las de desarrollo y progreso, y como dependiente de una actitud emprendedora a partir de la cual se configura el homo discentis; es decir, un sujeto en aprendizaje permanente y atento a las demandas del mercado (Noguera, 2011, citado por Loureiro y Lopes, 2019), cuestión que supone un desplazamiento de los propósitos humanistas de la educación en cuanto proceso de formación.

Lo que se advierte es la alta estima por el saber de información en detrimento de la formación como categoría pedagógica y educativa. De acuerdo con
Flórez (1998), la formación es principio y fin de la pedagogía y corresponde al proceso de humanización de los sujetos a partir de su imbricación en los procesos de educación y de enseñanza, la formación es lo que queda después de olvidar la información. El problema con la centralidad del aprendizaje es que este no garantiza la formación, pues como lo plantea el pedagogo, hay aprendizajes que no cumplen esa función con nadie y la suma de estos tampoco aseguran la formación. En este sentido, se parte de un marco en el que se problematiza el lugar de ella en el actual discurso educativo; de acuerdo con Nussbaum (2010), existe una crisis en cuanto a las humanidades y en cuanto al sentido humanista de la educación, panorama que advierte sobre el desvanecimiento de la formación en cuanto concepto básico en la historia del humanismo (Gadamer, 2012).

Del mismo modo, la formación, como concepto y práctica relacionada directamente con la cultura, no puede positivizarse. Por el contrario, exige el detenimiento y la reflexión acerca del sentido que adquieren los ejercicios de saber pedagógico en la escuela, específicamente desde la EF, no solo desde su acepción humanista, sino también en cuanto aprendizaje de la cultura, partiendo de la comprensión del significado o la acepción de la formación como un proceso constante de inculturación por el cual el ser humano apropia reglas, normas y condiciones, y se construye en la cultura en la cual se encuentra inmerso (González y Malagón, 2015).

Finalmente, el estudio se enmarca en el campo investigativo del pensamiento del profesorado y se inscribe en lo que Pérez y Gimeno (1998) denominan enfoque alternativo. En este sentido, se presta atención a los contenidos, las ideas y las teorías sobre la enseñanza y el aprendizaje, y se intenta trascender los análisis cognitivistas, a partir de reflexiones en las que se vinculan las relaciones entre la escuela y la sociedad, la cultura y el conocimiento, como factores que influyen en el pensamiento y la práctica del profesorado en el aula, explicaciones inscritas en un terreno más cercano a lo pedagógico. Lo anterior se enmarca, dadas las delimitaciones teóricas y conceptuales de este estudio, en el saber pedagógico, como saber propio del profesorado por cuanto es un saber, constituido y constituyente, por y de sus prácticas pedagógicas.

\section{Metodología}

El estudio se realiza desde un enfoque cualitativo y a través de la definición de una metodología en la que se privilegia la analítica interpretativa. Con esta configuración se toman aportes de diferentes métodos cualitativos, en virtud de la comprensión de los sentidos y significados sobre el objeto de estudio. Se parte de los postulados de Holstein y Gabrium (2013), 
quienes proponen una analítica nueva e híbrida de la construcción de la realidad (p. 245); una analítica del tipo bricolaje (Kvale, 2011) que, de acuerdo con Pinar et al. (1995, citado por Rifa, 2003), se inscribe en explicaciones posestructurales a través de las que se presta atención al lenguaje, el poder, el deseo y la representación como categorías discursivas.

Se privilegia la aproximación inductiva y se opta por la teoría fundamentada, en cuanto método, a partir del cual se fundamenta la generación de teoría afirmada en los datos sustantivos. La investigación adopta concepciones constructivistas e interpretativas, para propender por la generación y construcción de teoría a partir de la interpretación realizada sobre los datos de la base empírica, antes que por la confirmación o verificación de proposiciones teóricas establecidas a priori desde otros modelos de investigación (Stake, 2010).

\section{Diseño metodológico}

Se delimitó como población investigativa al profesorado en formación de EF. Específicamente, se seleccionaron ocho profesores en formación, atendiendo a los siguientes dos criterios: estar en curso el último año del programa de formación de licenciatura en EF y realizar la práctica pedagógica, práctica final o práctica profesional en el contexto escolar formal. La pesquisa recorre las siguientes fases, entre la recolección y el análisis de la información:

\section{Fase de contextualización analítica}

Corresponde a la revisión previa, o contextualización sobre lo que en la teoría del saber pedagógico se entiende como el archivo del saber pedagógico (orientaciones curriculares de las universidades participantes en el estudio, decretos sobre la formación del profesorado en Colombia, orientaciones curriculares para la EF escolar; es decir, una problematización previa básica del fenómeno). Este acercamiento permitió contextualizar la investigación en un marco que, en parte, definió o condicionó, en cuanto práctica discursiva, el saber pedagógico y los sentidos formativos que pudieran estar presentes en el profesorado en formación.

\section{Primera fase de recolección de la} información: filmación de la clase

Se filmó una clase desarrollada por cada uno de los profesores en formación. La clase que se observa se deja a la libre planeación de los sujetos de investigación.

\section{Primera fase de análisis: codificación abierta}

Una vez desarrollada y filmada la clase, se procedió a su transcripción y a un primer ejercicio de análisis con la ayuda del software Atlas.ti (versión 7.0). Desde este primer esfuerzo analítico, se realizó una codifica- ción abierta a partir de la lectura del video (se prestó atención al discurso, a los ejercicios, a los métodos, a las didácticas, a los saberes en práctica, a los estilos de enseñanza del profesor en formación, y en general, a la organización y desarrollo de la clase). Se analizó el saber pedagógico desplegado en la práctica pedagógica del profesor en formación observado.

\section{Segunda fase de recolección de} la información: la audiencia

La audiencia correspondió a un ejercicio de conversación o entrevista con los profesores en formación observados por universidad. A esta audiencia se llegó con la codificación abierta, la cual sirvió de base para la conversación. Motivo por el cual se diseñó una guía de entrevista semiestructurada que retomó aspectos claves surgidos del primer ejercicio de codificación abierta (análisis del video de clase), así como de la contextualización del estudio dentro del marco del saber pedagógico (archivo del saber pedagógico).

\section{Segunda fase de análisis: la codificación axial o selectiva}

Representa un "segundo momento" en el proceso de análisis. Se dio cuando ya existía una primera lectura de los datos obtenidos de la base empírica y representó la organización o clasificación de los códigos en familias de códigos.

\section{Tercera fase: análisis y codificación teórica}

A esta fase se llegó con la lectura analítica del video de clase y la audiencia con el profesorado en formación. Estos documentos primarios y su respectivo análisis fueron configurando, desde la solidez que puede permitir la recolección y análisis de la información, una ruta de codificación cada vez más sustentada en los datos. A partir de aquí, se volvió sobre las sesiones de clase observadas y filmadas, y se realizó un nuevo ejercicio de análisis; esta vez, a partir de los códigos que han emergido de la codificación abierta y de la codificación axial o selectiva, con la idea de consolidar el código sustantivo dentro de una explicación o síntesis conceptual a partir de un proceso de configuración teórica formal.

\section{Tratamiento de los datos y de la información}

El proceder analítico se dio simultáneamente con la fase de recolección de la información, atendiendo a los principios de la teoría fundamentada, específicamente a lo definido en su característica de método comparativo constante (Requena, Carrero y Soriano, 2006). El análisis se realiza a partir de dos grandes momentos:

- Codificación del material o información recolectada. Se realizó a través de la herramienta de análisis Atlas.ti (versión 7.0), proceso del 
que se salió con la emergencia de un código teórico luego de los procesos de agrupación de códigos en familias de códigos, en lo que representó un tránsito entre la codificación abierta y la codificación selectiva o axial sobre las diferentes fuentes de información (observaciones en campo, entrevistas, audiencias, análisis de videos de clase).

Las observaciones en campo y los análisis de video fueron realizados a partir de la aplicación de una rúbrica de observación construida por el equipo de investigación, a partir de la síntesis de elementos constitutivos del saber pedagógico condensados en las categorías: ser, saber (disciplinar y pedagógico), saber hacer (didáctica) y poder, instrumento validado a través de procesos de pilotaje y revisión de pares. Las entrevistas y audiencias se adelantaron a través de diseños semiestructurados y de conversaciones motivadas por elementos surgidos de las observaciones y del análisis de los videos de clase.
- Síntesis teórica. Se dio a través de un ejercicio de denotación/connotación del sentido formativo encontrado. Este proceso representó un esfuerzo por la construcción de una proposición teórica formal, soportada en los datos provenientes del análisis de las prácticas de saber pedagógico del profesorado observado. En esta fase, se recurrió a un ejercicio interpretativo a partir del análisis discursivo sobre los significados y sentidos captados en los procesos de codificación. Se trabajó un proceso de denotación y connotación del discurso, a partir de la exposición de fuentes primarias (texto o imagen) previamente codificadas, dado su carácter descriptivo y análogo (denotación) con códigos que condensaban su significado, para luego, en el ejercicio de connotación, presentar, de manera más visible, el mensaje captado como implícito. En la figura 1 se ilustra la forma en la que se realizó el análisis dentro del estudio.

A continuación, se presenta mediante un gráfico, la forma en la que se realiza el proceso de análisis dentro del estudio.

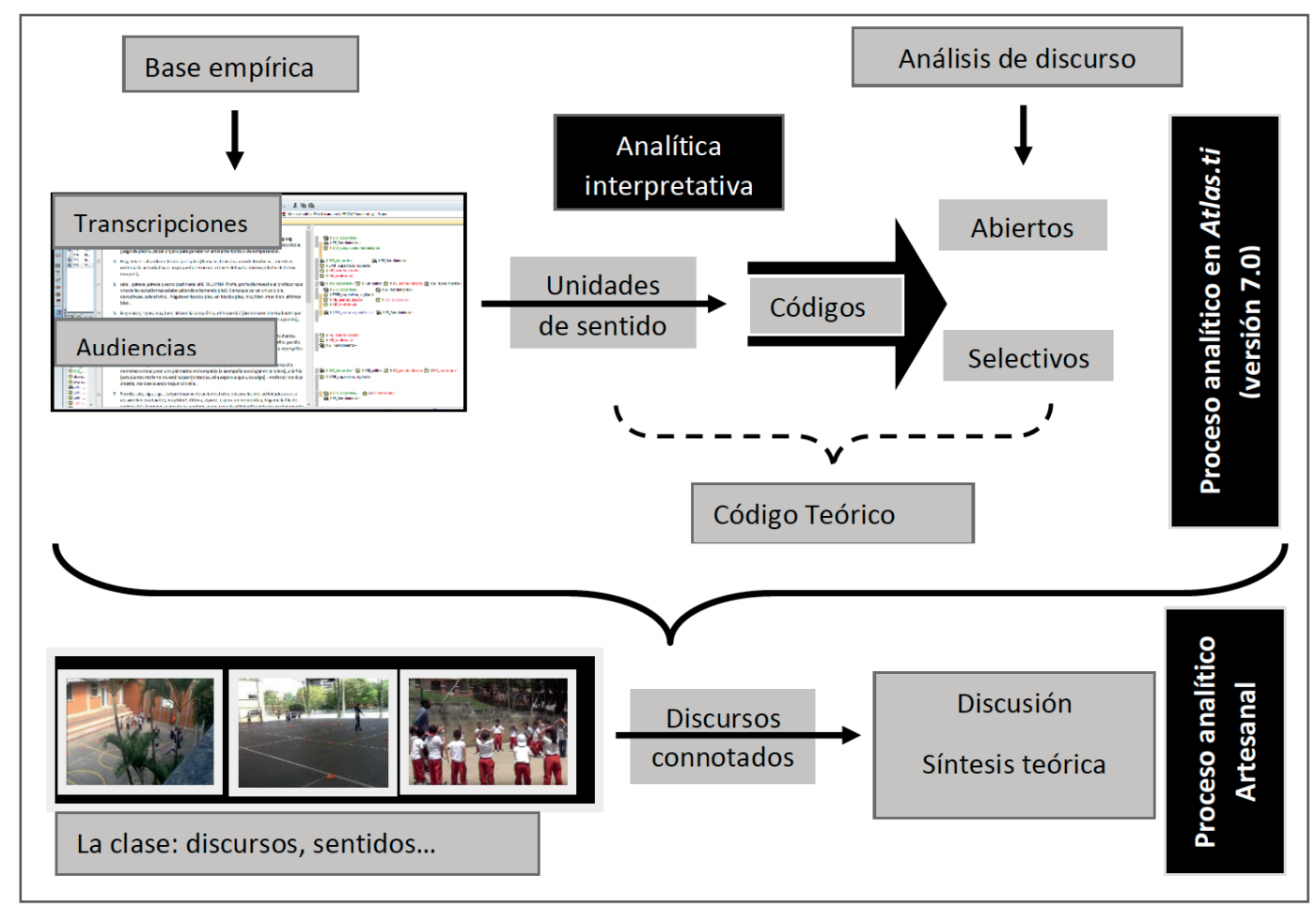

Figura 1. Proceso de análisis

Fuente: elaboración propia. 


\section{Resultados o hallazgos}

Los ejercicios de codificación abierta y la posterior fase de organización y categorización de los códigos en familias de códigos permitieron reconocer cuatro grandes familias de códigos: saber, interés, saber pedagógico y sentidos formativos. La configuración del rendimiento, en cuanto sentido formativo, obedeció a un ejercicio de análisis alrededor de los significados contenidos en las diferentes familias de códigos y en su interjuego, en cuanto prácticas discursivas o discursos en práctica desplegados en las intervenciones pedagógicas del profesorado de EF en formación.

Las aproximaciones a las fuentes primarias: filmación y observación de la clase (video), transcripción del video de clase audiencias y entrevistas con el profesorado en formación de cada universidad, dejaron reconocer, de entrada, la alta valoración que se tiene sobre el carácter competitivo como recurso metodológico y como componente didáctico en la EF escolar. Se logró evidenciar, en el terreno de lo práctico, así como mediante las entrevistas, un saber pedagógico caracterizado por una alta referencia al deporte, especialmente en su lógica de rendimiento, por encima de otros posibles enfoques o tratamientos como podrían ser el educativo o el recreativo. Esto se percibió en el reconocimiento de los intereses exclusivamente técnicos centrados solo en la ejecución motriz de la tarea, en la utilización de ejercicios específicamente

\begin{tabular}{|l|l|}
\hline & SF_Rendimien \\
\hline Discriminación por génerc & $n / a$ \\
\hline INT_Crítico & $n / a$ \\
\hline INT_Ecológico & $n / a$ \\
\hline INT_Práctico & $1-0,01$ \\
\hline INT_Técnico & $14-0,15$ \\
\hline SAB_Biomédico & $2-0,04 \quad 0$ \\
\hline SAB_Disciplinar & $5-0,06$ \\
\hline SAB_Enciclopédico & $4-0,07$ \\
\hline SAB_Sitaudo & 0 \\
\hline SAB_Técnico_Deportivo & $5-0,09$ \\
\hline SP_CURR_Funcionalista & $6-0,08$ \\
\hline SP_CURR_Interdisciplinar & $1-0,02$ \\
\hline SP_CURR_Recontextualizac & $n / a$ \\
\hline SP_DID_Competencia & $9-0,16$ \\
\hline SP_DID_deportiva & $11-0,17$ \\
\hline
\end{tabular}

físicos y con intencionalidades igualmente técnicas y coordinativas, y a través del reconocimiento de los ejercicios de retroalimentación realizados por los profesores observados durante sus prácticas pedagógicas, en los cuales se evidenció una figura del maestro cercana a la del rol de entrenador.

Estos elementos ayudaron a entender, en su conjunto, algunos aspectos configuradores del saber pedagógico, especialmente en lo que tiene que ver con las selecciones curriculares, el saber privilegiado, el interés proyectado con el saber, las metodologías y didácticas utilizadas, y las intervenciones y mediaciones pedagógicas durante la enseñanza. Lo anterior posibilitó la identificación de diferentes elementos que coinciden con la preocupación por la adquisición de habilidades exclusivamente procedimentales y motrices, e instaladas en una lógica de eficiencia, control y economía del movimiento, propia de la lógica deportiva y de la matriz biomédica, a partir de la cual se configura un profesor que, en su relación con el saber, se corresponde con la imagen de un ejecutor y aplicador de teorías y ejercicios fabricados por expertos ajenos al acto educativo.

Una mirada a los ejercicios de codificación, a través del software Atlas.ti (versión 7.0) facilitó reconocer cómo el rendimiento se entrecruza con códigos como el interés técnico, con una didáctica deportiva y competitiva y con unas metodologías tradicionales.

\begin{tabular}{|l|l|}
\hline & SF_Rendimien \\
\hline SP_DID_Juego & $1-0,02$ \\
\hline SP_EST_PROF_Acritico & $2-0,03$ \\
\hline SP_EST_PROF_Autoritario & $1-0,01$ \\
\hline SP_EST_PROF_Cálido & $1-0,01$ \\
\hline SP_EST_PROF_Conciliador & $n / a$ \\
\hline SP_EST_PROF_Critico & $n / a$ \\
\hline SP_EST_PROF_Reflexivo & $1-0,01$ \\
\hline SP_EST_PROF_Supervisor & $5-0,07$ \\
\hline SP_FBK_Militar & $5-0,07$ \\
\hline SP_FBK_Motivador & $1-0,02$ \\
\hline SP_MET_Constructivista & $n / a$ \\
\hline SP_MET_Descubrimientog & $n / a$ \\
\hline SP_MET_Tradicional & $12-0,17$ \\
\hline SP_PLAN_Flexible & $1-0,02$ \\
\hline SP_PLAN_Improvisado & $n / a$ \\
\hline SP_PLAN_Rígida & $n / a$ \\
\hline
\end{tabular}

Figura 2. Coocurrencia de códigos

Fuente: elaboración propia desde Atlas.ti. 
La figura 2 muestra, a partir del tono o intensidad del color verde, cómo el rendimiento presenta una co-ocurrencia con los demás códigos presentes en la base empírica del estudio.

En este sentido, se decanta una orientación implícita en las prácticas de saber pedagógico del profesorado de EF en formación, en la que el rendimiento va apareciendo como el fin o propósito último de la enseñanza; más allá de las explicaciones o pretensiones que se puedan tener previamente con la planeación. Se advierte una lógica en el tratamiento pedagógico que, en la práctica misma, configura un saber sujetado por el imperio de la motricidad en cuanto discurso que, en su explicación del movimiento humano, instalado en las razones de la ciencia, no deja margen a la trascendencia del acto de moverse. Esta cuestión encapsula a un profesor en las leyes del movimiento por el movimiento y que, dadas las condiciones prácticas y técnicas de la disciplina, se instalan fácilmente en la lógica del aprendizaje, en cuanto práctica educativa centrada solo en el qué y el cómo del ejercicio y privada de cuestionamientos como el porqué y para qué de lo que se hace; elemento que permite vislumbrar su despedagogización y despolitización de la enseñanza.

De acuerdo con lo anterior, llama la atención cómo el rendimiento, en cuanto sentido formativo se relaciona con los códigos anteriormente mencionados (interés técnico, didáctica deportiva y competitiva, y metodologías tradicionales), pero también con otros como un saber curricular funcionalista, un estilo profesoral supervisor y la utilización de un feedback (retroalimentación) militar. El tratamiento y relación con las selecciones curriculares, el estilo del profesorado y el tono (expresión verbal y no verbal) o cualidad de las intervenciones y retroalimentaciones durante la enseñanza también aportan a la delimitación o configuración del rendimiento como un sentido formativo implícito en el saber pedagógico.

Los códigos presentes forman parte de la discursividad que circula en las prácticas pedagógicas del profesorado en formación. Las palabras y acciones registradas y codificadas dan cuenta, en gran parte, de prácticas, conocimientos y representaciones que, en su conjunto, aportan en la configuración de un saber pedagógico caracterizado por la alta valoración del componente competitivo, del saber e interés técnico y de unas orientaciones tradicionales y deportivas. Estas son tratadas como recurso metodológico-didáctico y como contenido con propósito formativo desplegado en las intervenciones pedagógicas desde la EF en los patios escolares.

En la figura 2 se visualizan algunos de estos códigos, relacionados con las metodologías, las didácticas, los estilos de enseñanza y los estilos del profesor.
Todos estos códigos y las respectivas familias van perfilando la configuración de una intención o sentido formativo conectado con preocupaciones eficientistas que, a través de la depuración de las diferentes acepciones y de la discursividad alrededor del saber pedagógico de los profesores en formación observados, permiten develar una idea de formación por y para el rendimiento en la escuela y que, en este caso, es desplegada a través de las prácticas pedagógicas y legitimadas por el saber pedagógico del profesorado de un área escolar que parece no cuestionar los sentidos formativos, que desde esta discursividad, se producen y reproducen en la educación escolar.

Las diferentes citas vinculadas a los códigos y familias de códigos pueden estar directamente asociadas con el código rendimiento, pero también es posible que algunas de las citas. Al mismo tiempo, se encuentren asociadas a otros códigos, es decir, que sean concurrentes.

Tal y como se ha anunciado, el componente competitivo es uno de los elementos a partir de los cuales se configura una idea de rendimiento por parte del profesorado de EF en formación, la utilización de la competencia es quizá uno de los recursos a los que más se acude en la planeación, organización y realización de las clases. Así lo plantea uno de los profesores observados, quien otorga alto valor a este componente:

\footnotetext{
Me parece a mí que esta última [competitividad] es fundamental en estas edades y más en este grupo. El tema de la competitividad le va a dar mucha más motivación al estudiante durante la clase, y se puede ver ahí en el video que los niños siempre estaban...

“Hey!, cómo, vamos a ganar, vamos a ganar, vamos a ganar". (Comunicación personal entre profesores en formación 1 y 2, UdeA, 2019.).
}

En este sentido, la cita permite reconocer la forma en la que el profesor en formación, luego de su práctica pedagógica, avala y confirma el empleo o la inclusión del componente competitivo como medio didáctico y como elemento de motivación dentro de la clase. El profesor es quien define este elemento, que pudiendo ser intrínseco a algunos juegos, gana mayor relevancia en cuanto elemento privilegiado en la planeación y materialización de sus clases.

[...] que [eso de la competencia] es una estrategia o una herramienta bastante importante a la hora de dirigir la clase de educación física en la primaria porque es que los niños siempre están con la competitividad de por medio. Así yo logro centrar la atención en lo que estamos haciendo. (Comunicación personal entre profesores en formación 1 y 2 , UdeA, 2019). 
Me parece muy complicado diseñar una clase que sea "iVenga!, vamos a participar todos a la vez", “¡Venga!, vamos a hacer otra cosa, tal otra”. Mientras que, si está el componente de competitividad presente, la clase siempre va a estar... (chasquea los dedos), y en todas las clases siempre tengo el factor competencia presente y me ha ido muy bien con eso (Comunicación personal entre profesores en formación 1 y 2, UdeA, 2019).

Lo que se puede leer entre líneas es la introducción de una idea de rendimiento por la vía de la competencia y la competitividad dentro de la clase, no hay mayores reparos a este tratamiento. Lo que se observa, en cambio, es una confirmación de esta estrategia y a la vez, la clasificación o el señalamiento de los estudiantes entre ganadores y perdedores. También es sugestiva la manera en que el profesor en formación acompaña su argumento con la gestualidad referenciada en la cita; es decir, con la acción de chasquear los dedos, gesto que lleva un mensaje de eficiencia y economía del movimiento, es decir, de rendimiento.

De acuerdo con la anterior revisión de citas codificadas, se presenta un ejercicio de interpretación de los mensajes, significados y sentidos que circulan en las diferentes prácticas pedagógicas en cuanto espacio de visibilización del saber pedagógico del profesorado de EF en formación. La clase se convierte en un espacio-tiempo potente para la lectura sobre las intervenciones pedagógicas.

\section{Denotación y connotación del rendimiento en la enseñanza de la Educación Física}

A continuación, se presenta en un ejercicio de denotación/connotación el sentido formativo del rendimiento como una disposición u orientación que se halla implícitamente contenida en el saber pedagógico del profesorado de EF en formación y que, a la luz del análisis sobre las prácticas pedagógicas, se logra visibilizar en contexto, en tanto espacio-tiempo de materialización del saber pedagógico.

De acuerdo con Barthes (1986), la denotación obedece a un mensaje que es absolutamente analógico, es decir, que se encuentra privado de un código y que en su contenido refleja el acontecimiento tal como se está revelando; mientras que la connotación corresponde a la lectura del mensaje a partir de la imposición de un segundo sentido al mensaje propiamente dicho y representa la codificación del análogo, en este caso, como lo propone el autor, de la fotografía. En este estudio, se toma este principio para el desciframiento de los sentidos formativos implícitos en el saber pedagógico del profesorado de EF en formación, pero no solo a través de la fotografía sino de la clase misma, en cuanto espacio-tiempo capturado, en el que es posible analizar tanto lo que denota su contenido como lo que se puede connotar luego de la codificación a los diferentes documentos primarios analizados en las fases previas del estudio.

Tabla 1. Connotación del sentido formativo rendimiento

\section{ENUNCIADO DISCURSIVO}

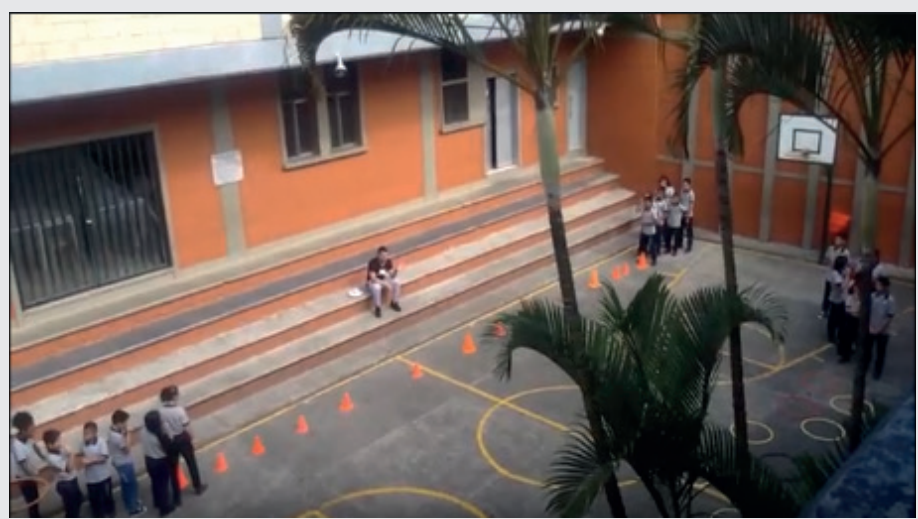

Fuente: video de clase, profesor en formación 1, UdeA, 2019.

Ciudad: Medellín. Institución Educativa Toscana. Clase de EF, 5ํㅜ, mixto. Universidad formadora del profesor: Universidad de Antioquia. 
La imagen denota a un profesor que, sentado en las gradas, dirige la actividad. También se observa al grupo de estudiantes dividido y ubicado en filas en las cuatro esquinas de la placa polideportiva del colegio. Sobre el suelo hay conos y aros.

Contextualización del enunciado. La foto corresponde a un momento en el que se utiliza un juego (piedra, papel o tijera) para dinamizar una actividad en la que los estudiantes deben realizar zigzag en velocidad por entre los conos y aros que hay sobre el piso del patio. El profesor en formación constantemente recurre a la competencia para motivar al grupo. El objetivo no es el juego de piedra, papel o tijera (juego popular), sino el ejercicio sobre las capacidades físicas.

Análisis descriptivo-comprensivo. La imagen en sí ya es bastante diciente: un grupo de estudiantes de primaria, dividido a su vez en cuatro subgrupos ubicados cada uno en hilera sobre las cuatro esquinas de una plaquita polideportiva gris. La representación es patética y conmovedora, obedece al escaso tiempo y espacio que le dedica hoy la escolarización al movimiento en la escuela; un movimiento que no solo por la captura o el congelamiento (obvio) de la foto, se advierte como un ejercicio de domesticación del cuerpo y del movimiento mismo. La línea recta de conos de demarcación, así como la sugestiva presencia de los aros sobre el cemento, que seguro está caliente en la inclemencia del ambiente de medio día en Medellín, sugieren pensar que lo que allí se da es mucho más que un juego, que la práctica moldea unas disposiciones que se desvanecen y se confunden, probablemente, en los deseos mismos del profesor que cómodamente orienta desde la tribuna. Sus razones o argumentos están tan acartonados de explicaciones académicas que parecen alinearse paralelamente a la línea que trazan los conos en el suelo, con la seguridad, casi incuestionable, del saber fisiológico y motriz que traza la matriz deportiva sobre la EF en la escuela. Poco a poco va saltando, sobre la imperturbabilidad de la fotografía o del momento capturado, la instrumentalización del juego popular y la domesticación de la cultura lúdica infantil presa de la ficción sacralizada del desarrollo técnico y motriz. ¿Cuántos, con el rótulo de "licenciados en Educación Física" nos atrevemos hoy en día a invertir la ecuación? A pensar al margen de las invenciones que ubicaron la EF en el lugar del cultivo y el compromiso motor y físico. La imagen lentamente revela el código contenido, como si dijera que toda esa organización y la utilización misma del juego de piedra, papel o tijera es solo un pretexto para que niñas y niños salten y sorteen obstáculos, no de juego, de entrenamiento. Es muy importante, advierte la icónica y el mutismo serio que ofrece el momento congelado. Juega, pero eficientemente. ¡Rinde!

ENUNCIADO DISCURSIVO

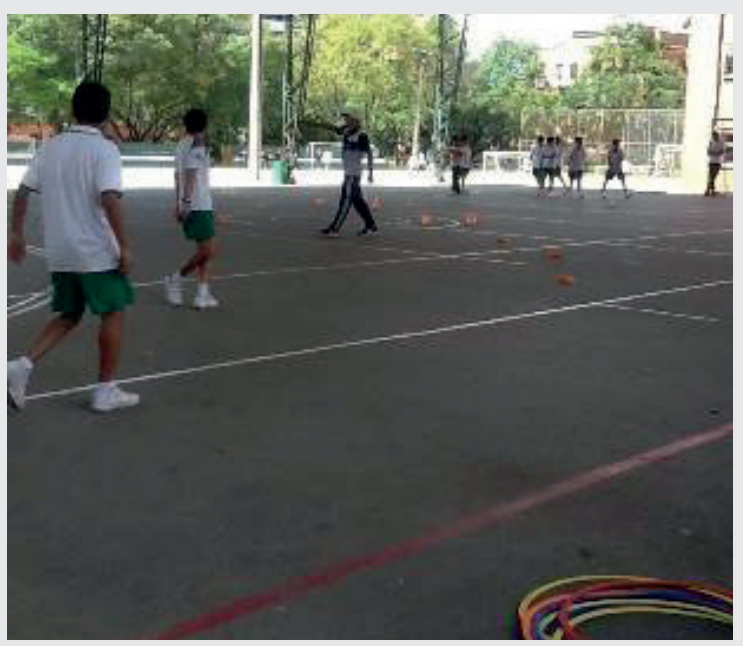

Fuente: Video de clase, profesor en formación 1, USB, 2019.

Ciudad: Envigado (municipio del área metropolitana al sur del Valle de Aburrá). Institución Educativa La Salle de Envigado.

Universidad formadora del profesor: Universidad de San Buenaventura. 
La imagen muestra una placa polideportiva en la que hay distribuidos, una cantidad de conos de demarcación, así como unos aros. El grupo de estudiantes se encuentra divido en subgrupos o equipos. En el centro, el profesor en formación organiza y dirige la actividad.

Contextualización del enunciado. El grupo de estudiantes que se encuentra en el patio o placa polideportiva ya ha sido dividido previamente en el salón; los hombres van a una práctica y las mujeres van a realizar otra con un profesor diferente. En la imagen se retrata la práctica realizada por los varones; se desarrolla una clase de juegos cooperativos, esa es la indicación que ha dado inicialmente el profesor. No obstante, ha sido reiterativo con la vinculación del componente competitivo dentro de la clase; se realizan juegos cooperativos desde actividades competitivas.

Análisis descriptivo-comprensivo. Ante la mirada desprevenida y también ante la mirada esperada de la matriz escolar y deportiva, la imagen representa, entre otras, el orden, el control y la certeza de una práctica estructurada. Los conos de demarcación son, nuevamente, protagonistas y faros dispuestos en el suelo para el adiestramiento del movimiento y de la expresión de los cuerpos. Todo parece estar calculado; quizá hemos pasado de la gimnasia a los juegos deportivos, pero la limpieza de la tradición higiénica de la EF queda descubierta en la fotografía. En la esquina descansan un grupo de aros coloridos, su destino es predecible en medio del contexto de la foto, la psicagogia y la psicomotricidad avalan bien su instrumentalizado y cosificado uso. La ciencia también respalda, con el pre-texto del desarrollo - una de sus metáforas favoritas- la conformación de un cuerpo dócil y productivo que en medio de la discursividad y los imaginarios circulantes no dejan espacio a la sospecha o interrogación: se trata del rendimiento por la vía del moldeamiento de cuerpos y subjetividades simétricamente deseadas. La cooperación, fin aparente y explicitado en la sesión de clase, se va desvaneciendo o perdiendo en la lógica oculta de un saber hacer caracterizado por la exaltación de las capacidades y condiciones físicas y privilegiado desde los inicios de la EF escolar. ¡Coopera y rinde!

\section{ENUNCIADO DISCURSIVO}

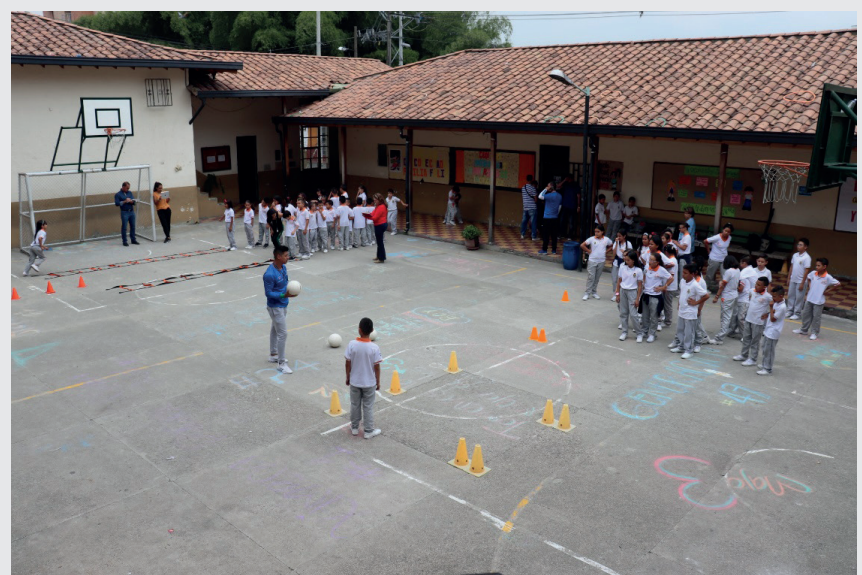

Fuente: audiencia profesores en formación 1 y 2, PCJIC, 2019

Ciudad: La Estrella (municipio del área metropolitana al sur del Valle de Aburrá). Institución Educativa Bernardo Arango Macías.

Universidad formadora del profesor: Politécnico Colombiano Jaime Isaza Cadavid.

La imagen muestra una placa polideprotiva en la que hay dos grandes grupos de estudiantes diferenciados, cada uno con un profesor y profesora diferente. El grupo del fondo hace clase con una profesora; se encuentran organizados en dos hileras, sobre el suelo dos escalerillas dispuestas para el desarrollo de la coordinación. El otro, el que sobresale en la foto, realiza clase con un profesor que sostiene una pelota en su mano derecha, el grupo de estudiantes observa con atención. Sobre el suelo de cemento gris se encuentran ubicados una serie de conos de demarcación deportiva. 
Contextualización del enunciado. La clase corresponde a una práctica en la cual se abordan fundamentos técnicos del fútbol; es una sesión en la que se enseña a cabecear el balón de forma perfilada. El profesor en formación argumenta que la clase tiene como objetivo trabajar la fuerza, la resistencia y la perfilación para cabecear y que, a su vez, se encuentra articulada a una unidad didáctica denominada "Juegos Predeportivos". Cada semana se aborda un deporte diferente (fútbol, baloncesto y voleibol).

Análisis descriptivo-comprensivo. En este caso, el sentido implícito es mucho más perceptible o se advierte de forma más visible. Los elementos privilegiados y la contextualización de la foto no dan entrada a la ambigüedad, como tampoco lo hace la seguridad manifestada a partir de la técnica, la economía y la eficacia del movimiento; estos parecen ser los fines perseguidos. Es recurrente esta relación de la EF con el entrenamiento deportivo en la escuela. Para muchos es lo esperado y es el fin último que justifica la presencia de la EF escolar; sus deseos se internalizan en la excusa del deporte escolar y educativo, pero se confunden cuando el énfasis trasciende de la experiencia al entrenamiento en cuanto ejercicio de profundización, terreno fértil para la lógica del rendimiento. Con esta práctica discursiva, el movimiento queda sujeto a los principios de un saber biomecánico y de una mecánica que ubica/condiciona el saber pedagógico del profesor en formación en la lógica del docente instructor, relación a partir de la cual la EF encuentra su sustentación en las leyes de la ciencia y el conocimiento fáctico en el que el profesorado es un sujeto supeditado a la aplicación de un saber probado y elaborado por otros. Así, la relación pedagógica por la vía de la EF escolar queda encasillada en los marcos seguros del saber calculado por la ingeniería curricular de la aprendificación de técnicas motrices encapsuladas en competencias y logros vertical y transversalmente asimilables. Mecánica de movimiento humano y curricular que perfilan la conformación corporal de un sujeto de rendimiento, la desprofesionalización del profesorado y la despedagogización de una EF deslumbrada por la cientifización del saber.

Fuente: elaboración propia.

La tabla 1 permite leer, en una primera aproximación sobre la imagen, algunas denotaciones, ya sea a partir de la foto como momento capturado/congelado, o desde la contextualización básica que se hace desde el video observado; foto y video representan, por su carácter análogo, un testimonio de la realidad por cuanto esta se revela fiel a su propio suceso. En este sentido, lo que denotan las tres imágenes es la preponderancia de una didáctica deportivizada; cada una obedece a una clase distinta, en una escuela distinta y desplegada por un profesor en formación igualmente distinto; formado, cada uno en una universidad diferente. Sin embargo, las semejanzas en la organización de la clase e incluso en los contenidos y medios seleccionados llaman poderosamente la atención.

En este sentido, desde esta síntesis, se pone el énfasis en el rendimiento como metáfora discursiva con poder sobre los procesos de subjetivación, a partir de las intervenciones pedagógicas desplegadas cotidianamente en los patios de las escuelas a través de las prácticas pedagógicas de la EF. Los planteamientos del posestructuralismo educativo ayudan reconocer la conexión entre saber y poder a partir de su enmarcación o configuración históricamente situada. Bajo esta premisa, Cherryholmes (1999) plantea:
A los docentes les gustaría creer que tienen control sobre lo que dicen y hacen, y que sus prácticas discursivas se basan en afirmaciones verdaderas. Pero si la verdad es discursiva y los discursos se hallan históricamente situados, no puede decirse la verdad en ausencia de poder, y cada disposición histórica de poder tiene sus propias verdades (p. 13).

El saber y el poder no podrían revisarse aisladamente en un análisis de las prácticas pedagógicas. Para Foucault (2002), saber y poder están directamente implicados uno al otro, y en ese sentido no existen relaciones de poder sin la constitución correlativa de un campo de saber, al tiempo que no puede existir un saber que no constituya unas relaciones de poder. No se puede analizar las relaciones saber/poder al margen de la práctica de un sujeto de conocimiento, en este caso, al margen del profesor en formación en cuanto sujeto de saber pedagógico, como si pudiera estar blindado frente al sistema de poder. Esta es la advertencia generada por el filósofo francés sobre la necesaria desnaturalización de las prácticas que, en contadas ocasiones, desplegamos bajo pretensiones de neutralidad o desde una ingenuidad (in)justificada frente a la ignorancia de las intervenciones pedagógicas. 
De esta manera, el rendimiento se configura como un sentido formativo implícito en el saber pedagógico de los profesores en formación de EF, vinculado con prácticas discursivas que encuentran asidero en la lógica escolar. En este caso, por la vía de las intervenciones corporales dispuestas desde las prácticas de saber de la EF. Este análisis también demanda pensar e interrogar la conexión entre el saber - disciplinar y pedagógico-y las prácticas pedagógicas, con los discursos que académica, institucional, cultural y socialmente circulan sobre la EF y de la educación escolar en general.

El filósofo coreano Byung Chul Han plantea que asistimos a la sociedad del rendimiento, sociedad caracterizada por la centralidad neuronal y por la positivización del mundo y de la vida. Esta cuestión define una nueva forma de entender y asumir el poder a partir del cual este pasa de una connotación negativa a una sugestiva positivización: el yo puedo, discurso que opera como regulador de las disposiciones individuales a partir del cual se crea un inconsciente social en el que se pasa del deber al poder, en cuanto disposición positiva del sujeto que se cree dueño de sí mismo y que, bajo esta ilusión, autogestiona su vida desde un afán de productividad y rendimiento que él mismo se ha impuesto (Han, 2012).

Asimismo, esa discursividad contenida en la sociedad del rendimiento guarda directa relación con las prácticas discursivas presentes en el saber pedagógico del profesorado de EF. Especialmente en lo concerniente a las didácticas utilizadas, así como a la predominancia de los intereses técnicos y del saber disciplinar que, sumadas con la tradicional y aún recurrente adscripción de la disciplina a la matriz biomédica, permiten reconocer unas disposiciones pedagógicas en la vía de la formación de subjetividades competitivas, individualistas y narcisistas más cercanas al eje del aprendizaje en cuanto educación de información, a partir del dominio de técnicas motrices, que a la preocupación formativa del eje educativo y pedagógico.

\section{Conclusiones}

Para entender las orientaciones pedagógicas, en términos de los sentidos formativos que configuran las prácticas de saber pedagógico, es necesario un análisis, no solo de lo que el profesorado declara o proyecta, sino de lo que se cristaliza en las prácticas pedagógicas como lugar de visibilización y materialización del saber pedagógico. Por ello, es indispensable abordar los contenidos, propósitos, métodos, didácticas, estilos de enseñanza y, en general, el tra- tamiento curricular que da el maestro como último curricularizador del saber durante las prácticas de enseñanza. A través del reconocimiento de estas diferentes dimensiones del saber pedagógico se reconoce el sentido que, explícita o implícitamente, se proyecta en las intervenciones corporales en la escuela.

La configuración del sentido formativo del rendimiento evidencia la persistente dependencia de la EF, como disciplina pedagógica, con el saber biomédico en cuanto referente para su definición disciplinar. De paso, establece una orientación con intereses técnicos en los procesos de enseñanza, elementos que permiten reconocer la propensión de la EF y del profesorado del campo, por una racionalidad aplicativa, lógica a partir de la cual la función pedagógica del profesorado se desvanece en proporción a su acercamiento con la disposición de un técnico ejecutor de saberes prediseñados externamente, contrario al ejercicio creativo y formativo de un profesorado portador de saber.

Asimismo, la alta valoración por el saber disciplinar por parte del profesorado en formación de EF permite reconocer la tendencia de la disciplina hacia una cientifización del saber. Este contexto viene perfilando un saber pedagógico enmarcado en razones y explicaciones técnicas en las que el tratamiento pedagógico, a partir o a través del movimiento humano, queda constantemente confundido en una preocupación exclusiva sobre el movimiento humano como problema u objeto de conocimiento. Tal diferencia sutil ayuda a entender la imposibilidad para trascender hacia un diálogo con la cultura de las prácticas, desplegadas desde y sobre el cuerpo, y con objetos propios del campo (el juego, el deporte, la recreación, la actividad física, las danzas, el ocio y el tiempo libre, etc.), en cuanto contextos de enseñanza y formación, más que como objetos de conocimiento e información. Dicha situación ha definido un profesorado preocupado exclusivamente por las técnicas motrices y el desarrollo motor, hecho que aleja a la disciplina y al maestro, en cuanto sujeto de saber, de una aproximación crítica con la cultura del movimiento, de las prácticas corporales y de los discursos que, en general, circulan en lo social con una alta incidencia dentro de la EF como materia del currículo escolar.

En ese sentido, se advierte una lógica más vinculada al paradigma del aprendizaje, como se ha presentado y señalado a lo largo de la discusión, que a una disposición afirmada en los terrenos de la formación. Esto debido a que el énfasis de las intervenciones pedagógicas y de las diferentes formas de manifestación del saber pedagógico del profesorado de EF en formación obedecen a la constatación inmediata 
y específica de habilidades y capacidades físicas, en particular respaldadas por explicaciones soportadas por ejercicios que, a través de los procesos de interpretación, logran ubicarse en la lógica del conocimiento científico en cuanto obedecen a prácticas aplicativas de saberes técnicamente validados.

En definitiva, se advierte que esta enmarcación del saber pedagógico dentro del eje del aprendizaje y la consecuente configuración del sentido formativo del rendimiento perfilan la formación, en cuanto disposición que finalmente perdura en el tiempo, de un sujeto alineado con la denominada sociedad del rendimiento, especialmente a partir de la discursividad alrededor del emprendimiento y de la positivización del mundo. Estos elementos aparecen constantemente imbricados con las ejercitaciones corporales desplegadas a partir del saber pedagógico del profesorado de EF en formación, prácticas discursivas asociadas a una cultura de la productividad y la eficiencia personal que terminan por internalizarse a partir de las intervenciones desde y sobre el cuerpo en la escuela. Lo anterior, respecto a las orientaciones superpuestas con las consignas del entrenamiento personalizado o el coaching, a partir de su vinculación, inicialmente corporal, y luego como toda una práctica vinculada con el aprendizaje continuo y con la consecución de objetivos, característica propia del sujeto de rendimiento, en cuanto disposición positiva para emprender un deber autoimpuesto.

\section{Referencias}

Aguayo, H. (2010). Las prácticas escolares de los educadores físicos. Perfiles Educativos, 32(128), 97-117. http://www.scielo.org.mx/pdf/peredu/v32n128/ v32n128a6.pdf

Barthes, R. (1986). Lo obvio y lo obtuso. Imágenes, gestos, voces. Barcelona: Paidós Comunicación.

Biesta, G. (2016). Devolver la enseñanza a la educación. Una respuesta a la desaparición del maestro. Pedagogía y Saberes, (44), 119-129. https://doi.org/10.17227/01 212494.44pys119.129

Carr, W. (1990). Cambio educativo y desarrollo profesional. Investigación en la Escuela, (11), 3-11. https:// idus.us.es/xmlui/bitstream/handle/11441/59257/ C a m bi oeducativoydesarrolloprofesional. pdf? sequence $=1 \&$ isAllowed $=y$

Cherryholmes, C.H. (1999). Poder y crítica. Investigaciones postestructurales en educación. Barcelona: Editorial Pomares-Corredor.
Díaz Barriga, Á. (2003). El currículo. Tensiones conceptuales y prácticas. Revista Electrónica de Investigación Educativa, 5(2), 1-13. http://redie.uabc.mx/vol5no2/ contenido-diazbarriga.html

Fernández-Balboa, J. (2003). Postmodernidad e investigación en la Educación Física. Ágora para la EF y el Deporte, (2-3), 5-22. https://www.researchgate.net/ publication/44385239_POSTMODERNIDAD_E_INVESTIGACION_EN_LA_EDUCACION_FISICA

Flórez, R. (1998). El fin de la historia o el fin de la pedagogía. Cuadernos de Pedagogía, (2), 17-26.

Foucault, M. (2002). Arqueología del saber. Buenos Aires: Siglo Xxi Editores.

Gadamer, H.-G. (2012). Verdad y método. Salamanca: Ediciones Sígueme.

González, H. y Malagón, R. (2015). Elementos para pensar la formación pedagógica y didáctica de los profesores en la universidad. Colombian Applied Linguistics Journal, 17(2), 290-30. https://doi.org/10.14483/udistrital. jour.calj.2015.2.a08

Gracia, A. (2008). La Educación Física en el mundo postmoderno. Corporeizando, 1(2), 171-183. http://revistas. pedagogica.edu.co/index.php/corporeizando/article/ view/674/664

Han, B. (2012). La sociedad del cansancio. Barcelona: Herder Editorial. http://raulkoffman.com/wp-content/ uploads/2012/07/Byung-Chul-Han-LA-SOCIEDADDEL-CANSANCIO.pdf

Herrera, G. (2017). Los discursos internacionales, la calidad de la educación y las políticas públicas. Educación y Ciudad, (33), 41-52.

Holstein, J. y Gabrium, J. (2013). Práctica interpretativa y acción social. En N. Denzin y Y. Lincoln (coords.), Manual de investigación cualitativa. las estrategias de investigación cualitativa (pp. 228-269). Barcelona, España: Gedisa.

Kvale, S. (2011). Las entrevistas en investigación cualitativa. Madrid: Ediciones Morata.

Loureiro, C. y Lopes, M. (2019). Aprendizagem: o imperativo de uma nova ordem econômica e social para o desenvolvimento. Pedagogía y Saberes, (51), 89-102. DoI: https://doi.org/10.17227/pys.num51-8054

Martos-García, D., Lorente-Catalán, E. y Marínez, J. (2018). Educación Física y pedagogía crítica: una necesidad educativa. En E. Lorente-Catalán y D. Martos-García (eds.), Educación Física y pedagogía crítica. Propuestas para la transformación personal y social (pp. 29-52). Valencia: Ediciones de la Universidad de Lleida. 
Nussbaum, M. (2010). Sin fines de lucro. Buenos Aires: Katz Editores.

Perez, A. y Gimeno, J. (1988). Pensamiento y acción en el profesor: de los estudios sobre la planificación al pensamiento práctico. Infancia y Aprendizaje, 42, 37-63.

Poblete, C. y Gamboa, R. (2013). Teorías implícitas y proyectos educativos: una visión paradigmática de la Educación Física. Educere, 17(58), 465-474. http://www. redalyc.org/articulo.oa?id $=35630404010$

Requena, A., Carrero, V. y Soriano, R. (2006). La construcción de la teoría a través del análisis interpretacional. Madrid: Centro de Investigaciones Sociológicas.

Rifa, M. (2003). Michel Foucault y el giro postestructuralista crítico feminista en la investigación cualitativa. Educación y Pedagogía, 15(37), 69-83.

Rojas, E. (2000). El hombre light. Una vida sin valores. Buenos Aires: Editorial Planeta.
Stake, R. (2010). Investigación con estudio de casos. Madrid: Ediciones Morata.

Stenhouse, L. (1987). La investigación y desarrollo del currículum. Madrid: Morata.

Tinning, R. (1996). Discursos que orientan el campo del movimiento humano y el problema de la formación del profesorado. Revista de Educación, (311), 123-134. http://redined.mecd.gob.es/xmlui/bitstream/handle/11162/71637/00820073003930. pdf? sequence $=1 \&$ isAllowed $=y$

Zapata, V. (2003). La evolución del concepto saber pedagógico. Su ruta de transformación. Educación y Pedagogía, 15(37), 177-184.

Zuluaga, O., Echeverry, A., Martínez, A., Restrepo, S. y Quiceno, H. (1988). Educación y pedagogía: una diferencia necesaria. Educación y Cultura. Revista del Centro de Estudios e Investigaciones Docentes de Fecode, (14), 3-9. 